\title{
La Création Et La Vénération Des Saints Au Maroc
}

\author{
Aguinou Lahoucine \\ Doctorant (GRAFE), Faculté des Sciences de l'Éducation- Rabat \\ Université Mohamed V, Maroc
}

Doi: 10.19044/esj.2018.v14n8p149 URL:http://dx.doi.org/10.19044/esj.2018.v14n8p149

\begin{abstract}
This article, which is part of social and religious relations, tries to study the sainthood and the cult of saints in Morocco. In order to achieve the objective of this work, we adopt an empirical method, which is based essentially on documentary research. To analyze the cult of saints in Morocco, this article presents, at first, the definitions of the words " sainthood " and " saint ". Then, the different appellations used by Moroccan people to designate a saint, and the types of existing saints in the Kingdom. Finally, the reasons for which these saints are created and venerated.
\end{abstract}

Keywords: Saint, religion, Morocco

Résumé

Cet article, qui s'inscrit dans le cadre des relations sociales et religieuses, tente d'étudier la sainteté et le culte des saints au Maroc. Afin de réaliser l'objectif de ce travail, nous adoptons une méthode empirique, qui se base essentiellement sur la recherche documentaire. Pour analyser le culte des saints au Maroc, ce travail présente, dans un premier temps, les définitions des mots « sainteté » et « saint » et les différentes appellations employées par les marocains pour désigner un saint. Ensuite, les types des saints existants au Royaume. Enfin, les raisons pour lesquelles ces saints sont créés et vénérés.

Mots-clés: Saint, wali, agurram, , agurrameté, marabout.

\section{Introduction}

Dans une ère où les sociétés musulmanes modernes inspirent les valeurs de la vie occidentale, caractérisées par la rupture entre le sacré et le profane (Skali, 2006). Les saints sont ubiquistes dans la vie de beaucoup de Musulmans, au point où chaque groupe et chaque localité a son propre saint vénéré. L'étude de la sainteté et le culte des saints au Maroc montre que les saints ne forment pas une typologie unique. Ils diffèrent selon les voies d'obtention de la sainteté. Ils jouissent des pouvoirs leur permettant 
d'accomplir les fonctions qui leur sont définit par la société. Dans cet article, nous tenterons de définir le «saint», et nous expliquerons pourquoi et comment les saints sont-ils créés et vénérés au Maroc? Pour approcher ces questions, nous adoptons une démarche empirique. Elle se base sur la recherche documentaire (ouvrages et articles de périodiques).

\section{La définition du « saint »}

Le mot « saint» est un terme appartenant au vocabulaire religieux chrétien. Dans le vocabulaire théologique de philosophie, il est définit comme un «être supérieur moralement, vénérable car vertueux, pur et parfait» (Jousset, 2009). Et « être saint est le résultat d'une vie spirituelle, là ou le sacré est une origine intouchable» (Jousset, 2009). Nous retenons dans ces définitions que le saint est une personne pure et idéale différente des autres, consécutive d'une vie spirituelle.

Dans le cadre de ce travail, le terme "saint» est utilisé comme équivalant du terme "marabout», employé par certains chercheurs occidentaux, et les termes employés par la société marocaine, arabe et berbère, qui sont : «cheikh», "wali », «chrif», « saleh », et «agurram ».

La définition du mot «saint» nous renvoie à la définition du mot amazigh " agurram », utilisé par les berbères, avant l'arrivée de l'Islam au Maghreb, pour désigner une personne connue par sa sainteté. Généralement, un «agurram» chez les amazighes, est une personne choisie par Dieu. Elle possède la qualité de ce que Gellner a appelé «l'agurrameté », qui signifie «la sainteté », dont l'essence est la médiation divine. En dialecte arabe marocain, il est appelé « charif» ou, tout simplement, «chrif».

Pour appeler un «saint», les marocains emploient les locutions suivantes : "sidi», ou «sayidi», d'où les appellations «Sidi Hmad Ou Moussa », par exemple. La locution « sayidi» veut dire : mon « seigneur ». Elle ne s'adresse qu'aux personnages honorables (Doutté, 1900). Les populations marocaines arabes emploient le mot «chrif» et ils l'appellent "moulay », nous citons l'exemple du plus ancien saint de Fès et du Maroc appelé : «Moulay Driss».

Quant aux femmes, que rien ne les empêchent de devenir des saintes, une fois acquises ce titre, elles sont appelées « lalla». Dans ce cadre, Doutté affirme que, «dans l'Afrique du Nord l'appellation la plus honorifique pour une femme est lalla «لالة لإل, «madame », qui est appliquée à toutes les saintes. C'est sans nul doute un mot berbère » (Doutté, 1900). À la région de Souss, nous citons l'exemple de la sainte Lala Tâ' $a z a$, à la province de Tiznit. 
Quant aux appellations «marabout», ou « maraboug ${ }^{25} »$, employées par certains chercheurs occidentaux, pour signifier « le saint », il nous semble qu'elles représentent des altérations du mot arabe «رابط

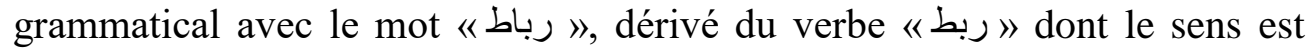
«lier». Les personnes attachées à l'endroit saint sont appelés «morabitin » ou simplement «mrabtin », qui veut dire : les liés et les attachés.

Concernant le nom « wali », il fait partie de la liste des quatre vint dix neuf noms divins, utilisé à de nombreuses reprises dans le Coran. Généralement, c'est celui dont l'affaire est prise en charge par Dieu. Il est un ami de Dieu, qui l'aime et le protège, comme l'explique le Hadith du Prophète: «Dieu le Très-Haut a dit: Je déclarerai la guerre à quiconque se montre hostile à l'un de Mes amis (walī)» ${ }^{26}$.

\section{Comment les saints sont- ils créés ? Quels sont leurs types ?}

En Islam, la genèse et l'évolution de la tradition religieuse et spirituelle inspire ses fondements de l'héritage prophétique, qui se transmet depuis le Prophète, selon des voies légitimement reconnues.

Au maraboutisme, toute personne, sans distinction, ni de race, ni de caste, peut devenir un saint, par plusieurs voies. Dans ce sens, E. Doutté a écrit : « la science, les bonnes œuvres, la réputation de justice, l'ascétisme, les pratiques mystiques, la folie et même l'imbécillité, peuvent conduire à la dignité de marabout» (Doutté, 1900). Les saints peuvent être vivants ou morts. Quelques-unes des qualités précitées ne permettent pas la possession du titre de saint, qu'après la mort, comme l'explique Doutté : « À défaut de la naissance, le savoir, les bonnes actions, la charité, la droiture du caractère, peuvent conférer à celui qui les possède le titre de marabout, mais seulement, dans ce cas, après sa mort » (Doutté, 1900). Et à propos des qualités qui permettent la possession de ce titre avant la mort, ce chercheur affirme que :

Pendant la vie n'est marabout que celui qui, descendant du Prophète, détient par son intermédiaire une parcelle de baraka, ou celui qui par des signes extérieurs non équivoques, a révèle comme particulièrement favorisé de la grâce divine au nombre de ces signes extérieurs sont la folie, l'extase, le don des miracles. Les fous, en effet, les idiots, et ceux qui se donnent. (Doutté, 1900).

La typologie du saint dépend du pouvoir charismatique de chacun et de l'environnement dans lequel il s'insère, des représentations et des croyances populaires, dont il fait l'objet. C'est ainsi qu'elle est déterminée par la voie suivie pour obtenir la sainteté, la généalogie, la perception de la légende

\footnotetext{
${ }^{25}$ Cette appellation a été employée par le Baron de $\mathrm{S}^{\mathrm{t}}$ amont ambassadeur français au Maroc en 1696, et François saint-Olon, l'ambassadeur du même pays au Royaume à la fin du 18 è siècle.

${ }^{26}$ Ce Hadith est qodsi. Il est rapporté par Al-Bokhari.
} 
et de l'histoire, la vie ou la mort, le pouvoir symbolique, et le pouvoir spatial du saint. Il est possible de vénérer des personnalités savantes, folles, mendiantes, etc.

Dans ce sens, Attirmidi a distingué deux voies de la sainteté. La première passe par 1'exercice spirituel. La seconde n'est pas mystique, forcement, mais elle permet l'obtention de la qualité du saint, sans effort, par la connaissance des secrets du monde et des créatures divines. Cette idée est confirmée par Ibn Khaldoun, qui disait que les fous, privés de leur capacité de vivre en société, tenant un rapport, permanent, à Dieu, peuvent obtenir la sainteté $^{27}$.

En général, les saints « morts » ou «vivants », se diffèrent selon la situation sociale, le niveau intellectuel de la société, et l'influence du pouvoir politique. Parmi eux, ceux qui sont des personnages « religieux » ou « laïcs », « effectif» ou « fictifs », « actifs » ou « inactifs », et « obscurs », ou « apparents ».

$\mathrm{Au}$ premier niveau, le saint héréditaire du prestige religieux de ses ancêtres, cheikh d'une zaouïa vénéré. Le pouvoir de ce dernier est souvent plus important que le pouvoir d'un agent politique, dépassant le niveau local. Il acquit le statut, appelé par Jamous « le saint régional » (Jamous, 1995). Puis, par des degrés successifs de sainteté, on descend du grand saint jusqu'au petit, dépendant pour survivre des charités des fidèles, et ayant son lieu à proximité du tombeau d'un ancêtre saint.

La voie d'obtention de la sainteté est déterminante de la typologie des saints. C'est ainsi que la voie de la science crée des saints érudits et « sérieux ». La voie des bonnes œuvres, la réputation de justice, l'ascétisme, les pratiques mystiques, la folie et l'imbécillité produisent des saints légendaires, appelés, par Dermenghem (Dermenghem, 1982), « populaires » ou «folkloriques », qui sont souvent des saints «locaux», et des saints « villageois », dont le pouvoir ne dépasse pas le niveau local. Les légendes de ceux-ci sont souvent difficiles à percevoir. Les saints ne sont pas tous neutres et non violents. Il arrive que certains d'entre eux soient tués en martyr, et obtiennent le statut du « saint martyr », juste après leurs disparitions.

\section{Pourquoi les saints sont-ils créés et vénérés ?}

La sainteté est une donation divine. Elle traverse les temps et les espaces. Elle influence les circonstances et l'environnement où elle se manifeste. Les saints sont des personnages fonctionnels et exceptionnels. Ils assurent des rôles temporels et symboliques qui leur sont définis par la société. Leur neutralité leur permet de jouir pleinement de leurs autorités. Selon Gellner, ils sont des leaderships légitimes. Ils gèrent les problèmes et les

\footnotetext{
${ }^{27}$ Cette dernière voie semble être beaucoup plus supérieure pour les soufis.
} 
conduisent vers la réalisation des objectifs, et servent de cœur d'appel dans le règlement des conflits. Ils fournissaient la logistique et des témoins, nécessaires, au serment collectif. Ils assuraient la supervision et la ratification des élections. Ils établissaient et garantissaient les liens intertribaux et les arrangements de jumelage de familles, dans des tribus différentes, dans le but d'échanger l'hospitalité en relation avec le commerce. La protection des voyageurs et la garantie des liens commerciaux et autres activités intertribales, étaient parmi leurs fonctions. Ils veillaient au respect du statu quo intertribal, notamment, dans les frontières territoriales complexes, et les droits de pâture qui se limités en temps et en espace (Gellner, 2003). Leur positionnement leur permettait, également, de servir de bureau de placement pour ceux qui sont forcés de chercher une nouvelle place au sein de la structure tribale. Ils sont capables d'ancrer le pouvoir sur le terrain et de tenir des alliances avec les acteurs politiques actifs. Leur sainteté assure le respect de leurs décisions (Doutté, 1900). Ces personnages fournissent au système religieux une raison d'être, en tant que réformateurs. Ils assurent l'encadrement et le conseil des fidèles, et la réponse à leurs questions relatives à la pratique religieuse. Ils interprètent des textes et adaptent leurs teneurs aux spécificités locales, comme l'affirme Gellner : « en somme, leur rôle se réduirait à fournir une assistance techno-religieuse à la maintenance et au management des structures établies » (Gellner, 2003). Cette représentation est, également, soutenue par Doutté qui a écrit : «Enfin, ils faisaient pénétrer quelque instruction dans les têtes dures des Berbères » (Doutté, 1900). Les saints présentent le modèle islamique, qui dispense "l'éducation de l'âme », recherché par les fidèles (Boubrik, 2012).

En plus des fonctions précitées, les saints ont des fonctions socioéconomiques, leurs permettant d'influencer la vie quotidiennes des disciples. Le cumul des dons et des récoltes apporté par les fidèles leurs permet d'honorer leurs engagements envers les différents acteurs de leur environnement. Les institutions qui leur sont attachées constituent, jusqu'à nos jours, des centres de redistribution des richesses et de satisfactions des besoins des serviteurs et visiteurs. Les terrains sont exploités contre une part de la récolte versée, annuellement, au dépôt du saint (Hammoudi, 1980, 627).

À côté de ces rôles et fonctions, les saints jouissaient des pouvoirs symboliques et politiques. Leurs autorités se concrétisent par plusieurs capacités. Ils dispensent la baraka et invoquent la bénédiction divine sous forme de prospérité, guérisons, et miracles (Gellner, 2003, 86), ou faire du mal en faisant agir la malédiction. Ils performent les miracles, et possèdent des pouvoirs magiques (Gellner, 2003, 82). Ils produisent des prodigues qui passent souvent par la magie, l'utilisation des talismans, et la prononciation des formules magiques pour traiter les patients, féconder la terre et les animaux, etc. Dans ce cadre, Montagne a remarqué que les saints héréditaires 
représentés au Maroc par les chorfas et igurramens, ne possèdent pas cette force, même si « leur simple présence suffit pour rendre un pays prospère » (Montagne, 2013, 87).

$\mathrm{Au}$ niveau politique, le positionnement des saints leur procure une place primordiale dans la gestion de l'affaire politique, même, si certains chercheurs, tels Gellner et Bardouzi, pensent que le maraboutisme est neutre par rapport au politique, et que d'autres, tels Geertz et Rabinow, pensent que la plupart des saints combinent des proclamations de fausse neutralité et l'ambition politique. Généralement, la qualité du saint ne dépend pas du sacrifice de la vie, mais de la bénédiction qu'il procure aux fidèles, mais il arrive que certains martyrs aient obtenu le statut du saint.

Certes, les saints exercent une domination sociale, mais seulement, si la population croit à leur sainteté (Gellner, 2003, 150). En soutenant cette thèse Berdouzi affirme que : « l'autorité et le leadership des saints est seulement une affaire d'apparence. Ils suivent, alors qu'ils semblent être suivis » (Berdouzi, 2012, 95). Cette relation d'interdépendance existant entre le saint et ses fidèles, témoigne du fonctionnement de l'échange symbolique, conditionné, liant les deux parties : le dominant et le dominé. Bourdieu, explique les conditions dudit échange, et le rapport de domination symbolique qui en résultent :

Il faut que les deux parties aient des catégories de perception identiques. Et cela vaut aussi pour les actes de domination symbolique qui [...] s'exercent avec la complicité objective des dominés, dans la mesure où, pour qu'une telle forme de domination s'instaure, il faut que le dominé applique aux actes du dominant (et à tout son être) des structures de perception qui soient les mêmes que celles que le dominant emploie pour produire ces actes. La domination symbolique (c'est une manière de la définir) repose sur la méconnaissance, et donc la reconnaissance des principes au nom desquels elle s'exerce (Bourdieu 1994, 187).

Pour obtenir la bénédiction du saint, le fidèle doit accomplir une série de conditions, lors de l'exercice des rituels. Ces conditions se limitent en trois éléments essentiels : le lieu, le temps, et les actes de dévotion (Gaborieau, 1994, 89-94).

\section{$\checkmark \quad$ Le lieu}

Dans toutes les religions, le lieu du culte occupe une grande place. En Islam, l'espace est désacralisé. La contrainte spatiale ne se pose pas. Les rites peuvent être pratiqués dans n'importe quel lieu, à condition qu'il soit purifié. Quelques rites limités ne se pratiquent que dans des lieux bien déterminés ${ }^{28}$.

${ }^{28}$ La contrainte spatiale se pose en Islam dans les rites suivants : La prière du vendredi, la prière des deux grandes fêtes de Rupture du jeûne et du Sacrifice, les prières spéciales pour les temps de sécheresse, les éclipses, et le pèlerinage. 
La caractéristique, la plus frappante, du culte des saints au Maroc réside dans le fait de multiplication des lieux sacrés, qui font concurrence aux trois lieux canoniques de l'Islam, appelés : «hurums $\|^{29}$. Les espaces regroupant toutes les institutions et tous les acteurs attachés au saint, directement ou indirectement, forment les «hurums du saint». Ils symbolisent des lieux centres de ce culte, qui sont en général:

Les tombes où sont enterrés les saints puis par extension les lieux qui ont été associés des événements de leur vie, comme les grottes où ils ont séjourné les endroits où ils ont fait étape et ceux qui contiennent leurs reliques (Gaborieau, 1994, 90).

Les tombes des saints sont, habituellement, enterrées dans des lieux isolés, à l'intérieur ou à l'extérieur des cimetières. Ces lieux d'enterrement sont, souvent, indiqués par les saints eux-mêmes, dans leurs vies, ou par leurs héritiers ou leurs fidèles. Ils peuvent être, également, désignés par ceux-ci, après leurs morts, en l'indiquant à l'un des fidèles vivants, dans le rêve ou par la vision. Généralement, ces tombes attirent des pèlerins pour des raisons sociales, thérapeutiques ou religieuses (Rhani, 2009, 27). Au Maroc, les descendances des saints construisent des dômes sur les tombes de leurs aïeuls, ce qui assure la protection des statuts des ancêtres, et subséquemment les leurs (Naji, 2011, 96).

La révérence des lieux saints se manifeste à travers le déplacement, l'orientation des fidèles vers ces lieux, et à travers les circumambulations qui se font sur place.

Le déplacement vers ces lieux pour les vénérer constitue une concurrence ou une imitation au vrai pèlerinage canonique $\left\langle a l-h a j »^{30}\right.$. Ce dernier n'est pas un simple voyage, mais une préparation au voyage absolu pour l'au-delà. Cette pratique condamnée par les opposants du culte des saints, est considérée légale, par ses souteneurs, qui pensent qu':

Il n'est pas interdit de faire des visites (ziyârât, singulier ziyârd) sur les tombes des saints, si l'on ne fait pas de pèlerinage spécifiquement dans l'intention de s'y rendre, et si les actes de dévotion qu'on y fait restent dans des limites permises (Gaborieau, 1994, 92).

Quant à l'orientation vers les lieux saints et la circumambulation sur les tombes en Islam, il n'est admissible que de s'orienter vers une seule et unique direction. Il s'agit de la direction de la Mecque et plus particulièrement

\footnotetext{
${ }^{29}$ Les lieux sacrés en Islam sont : al-haram al-macqui, lieu du pèlerinage à la Mecque, lieu de vie et d'enterrement du Prophète à la Médine, et Jérusalem, lieu du voyage nocturne et de l'ascension du Prophète.

${ }^{30}$ En arabe le mot «al-haj- الحج), veut dire le «pèlerinage ». Les opposant du culte des saints n'emploient pas ce terme pour exprimer les visites des fidèles au tombeau du saint, car ce mot est réservé au voyage sacré vers la Mecque pour vénérer les hurums de l'Islam, au moins une fois dans sa vie si on en a les moyens.
} 
le sanctuaire de la «Ka'ba- الكعبة" 》. Il est donc, strictement, interdit de se tourner vers les tombes des saints pour les vénérer, ou de faire la circumambulation «attawaf - الطو اف》, autour d'elles. Il est, également, interdit de se prosterner devant des créatures vivantes ou mortes, ou de se tenir debout devant elles pour faire des prières, car cela est réservé à Dieu.

\section{$\checkmark \quad$ Le temps}

Le temps a la même importance que l'espace dans le culte des saints en Islam. Il est un élément fondamental dans la pratique des rites quotidiens, au point où il a construit son propre calendrier lunaire. Les temps des rites sont codifiés avec précision (Gaborieau, 1994, 89).

Pour les puristes musulmans, fixer un moment déterminé est un privilège réservé à Allah, pour les rites canoniques. Au maraboutisme, les fidèles réservent un jour déterminé pour visiter la tombe de leur saint (Gaborieau, 1994, 90). Généralement, le moussem est une célébration collective annuelle qui renforce les pouvoirs spirituels du saint et des lieux sacrés qui lui sont attachés. Il s'organise à une datte calendaire établie, pour vénérer le wali vivant ou mort. La datte fixée pour ce rendez-vous est souvent le jour de l'anniversaire du saint. Pendant cette fête les foules des fidèles se regroupent dans les sanctuaires construits autour de sa tombe.

Les rituels pratiqués lors de cette célébration sont interdit par le Wahhabisme qui s'appuie sur les écrits d'Ibn Taymiya.

\section{$\checkmark \quad$ Les actes de dévotion}

Nous étudierons les actes de dévotion aux lieux saints en les répartissant en trois principaux éléments qui sont : Les gestes, les paroles et les offrandes

\section{- Les gestes}

Chez les musulmans, la gestuelle occupe une grande place dans leurs différentes pratiques religieuses. Les gestes effectuées lors desdites pratiques ne sont admissible que s'elles respectent les règles fixées. Leur modification est strictement interdite, et le modèle idéal est encadré par le conseil du Prophète qui disait : «Priez comme vous me voyez prier! » ${ }^{31}$.

\section{- Les paroles}

Le rituel du culte des saints emploie des paroles pour demander les faveurs et la bénédiction des personnages vénérés. Ces paroles sont libres. Elles peuvent être utilisées en autres langues que l'arabe, la langue du livre sacré, utilisée pendant les prières. Les paroles s'adressent au saint, sous forme

${ }^{31}$ Ce Hadith est authentique. Il est rapporté par Al -Bokhari dans son recueil. 
de serments ${ }^{32}$. En contrepartie de la bénédiction «la baraka » et des faveurs que le fidèle demande, celui-ci fait des promesses au wali. Pour les puristes ${ }^{33}$, le musulman n'a pas le droit de demander des faveurs qu'à son fondateur. Ces rites doivent, donc, être réservés à Dieu.

Les saints récitent des paroles divines afin de s'approcher de Dieu. Ces paroles leur permettent, également, de produire des effets miraculeux. Les fidèles de leur part, récitent des milliers de fois, de Noms divins, de formules coraniques ou de prières litaniques, en vue d'obtenir la bénédiction.

Les paroles comme acte de dévotion, sont plus importantes que les donations, chez les adeptes du culte des saints au Maroc. Elles peuvent remplacer les donations pour les fidèles pauvres, comme l'affirme Bourgeois : « Si on n'a rien à donner, on peut réciter une prière, ce qui équivaut à l'aumône »(Bourgeois, 1960, 10).

\section{- Les offrandes et les sacrifices}

Les tombes des saints et les institutions qui leurs sont attachées accumulent des offrandes et des donations, apportées par les disciples désireux de purifier leurs richesses et d'obtenir la bénédiction. Ces sacrifices sont des vertus préventives qui empêchent la malédiction et assurent la baraka et la réussite des projets, quels soient. Les sacrifices sont enrichis par des dons de toutes natures. Certains fidèles font même des sacrifices sanglants au nom des saints, sur leurs tombes. Afin d'obtenir la bénédiction, les donations et les sacrifices offerts doivent être de meilleure qualité. Pour cette raison, « On doit toujours offrir ce qu'on a de meilleurs. On ne doit pas donner un billet déchiré en aumône ; il faut choisir de préférence un billet neuf » (Bourgeois, 1960, 11). Quant aux sacrifices sanglants, l'animal sacrifié doit être de bonne qualité. Les fidèles apportent une part de leurs productions annuelles et présentent des aumônes comme sacrifice, au nom de Dieu ou du saint. Ces richesses amassées doivent être utilisées pour le bien. Pour les opposants de ce culte, ces sacrifices sont inadmissibles.

\section{Conclusion}

Le saint est une personne différente des autres. Il est fonctionnel et exceptionnel. Il assure des rôles temporels et symboliques qui lui sont définis par la société. Il est équivalent dse termes: marabout, cheikh, wali, chrif, saleh, et agurram. Ce dernier est employé par les berbères avant l'apparition de l'Islam, pour désigner une personne choisie par Dieu, possédant la sainteté.

\footnotetext{
${ }^{32}$ Le serment liant le disciple à son cheikh est un pacte liant le maitre à son fidèle disciple.

${ }^{33}$ Les puristes désirent réformer les pratiques religieuses des communautés. Leur courant vise d'amener les gens à devenir de bons musulmans, en abandonnant les pratiques interdites par la religion.
} 
Au maraboutisme, toute personne, peut devenir un saint, par plusieurs voies. Certaines qualités comme la science, les bonnes œuvres, la réputation de justice, l'ascétisme, les pratiques mystiques, peuvent conduire à la sainteté. D’autres telles le savoir, les bonnes actions, la charité, la droiture, permettent la possession du titre de saint, après la mort.

En général, les saints se distinguent selon leurs pouvoirs. Ils peuvent être : « effectif » ou « fictifs », « actifs » ou « inactifs », et « obscurs », ou « apparents ».

Les saints accomplissent des fonctions multiples, et jouissent des pouvoirs temporels et symboliques, leurs permettant d'influencer la vie quotidienne des fidèles. En contrepartie de leur bénédiction et leur protection, ces derniers leur apportent des dons et des sacrifices.

\section{References :}

1. Berdouzi, M. (2012). Structures et dynamiques sociales au Maroc: Évaluation des analyses anglo-américaines. Casablanca: Les Éditions La Croisée des Chemins.

2. Boubrik, R. (2000). Fondateur et héritiers. La gestion d'une succession confrérique (Mauritanie). Cahiers d'études africaines, 159, 2000, mis en ligne le 15 octobre 2000, 433-466. En ligne http://etudesafricaines.revues.org/25.

3. Bourdieu, P. (1994). Raisons pratiques. Sur la théorie de l'action. Paris: Seuil.

4. Doutté, E. (1900). Notes sur l'islâm magribin : les marabouts. Paris: Ernest Leroux, Éditeur.

5. Gaborieau, M. (1994). Le Culte des saints musulmans en tant que rituel : controverses juridiques / The Cult of Moslem Saints as a Ritual: juridical Disputations. Archives de sciences sociales des religions. V. 85, $\mathrm{N}^{\circ} 1,85-98$. En ligne

6. http://www.persee.fr/doc/ assr_0335-5985_1994_num_85_1_1427.

7. Gellner, E. (2003). Les Saints de l'Atlas (trad. P. Coatalen, intro. Gianni Albergoni). Paris: Bouchêne, Collection «Intérieurs du Maghreb ». (Oeuvre originale publiée en 1969)

8. Hammoudi, A. (1980). Sainteté, pouvoir et société : Tamgrout aux XVIIe et XVIIIe siècles. Annales. Économies, Sociétés, Civilisations. 35e année, $\mathrm{N}^{\circ}$ 3-4, 615-641. En ligne

9. http://www.

persee.fr/doc/ahess_03952649_1980_num_35_3_282657].

10. Jamous, R.(1995). La fabrication des saints : Faire, défaire et refaire les Saints, les Pir chez les Méo (Inde du Nord). Terrain (Carnets du patrimoine ethnologique), $\mathrm{N}^{\circ} 24,43-56$. En ligne

11. http://terrain. revues.org/3114]. 
12. Jousset, D. (2009). Le vocabulaire théologique en philosophie. Paris: Ellipses Edition.

13. Naji, S. (2011). Fils de saints contre fils d'esclaves : Enquête au cour du patrimoine immatériel marocain. Rabat: Edition DTGSN.

14. Paul, B. (1960). Charité et hospitalité, l'amour du prochain. L'univers de l'écolier marocain, fascicule $n^{\circ} 4$. Rabat: Ministère de l'éducation nationale, de la jeunesse et des sports, Faculté des Lettres et des Sciences Sociales.

15. Rhani, Z. (2009). Le chérif et la possédée. Sainteté, rituel et pouvoir au Maroc. L'Homme, Éditions de l'EHESS, 2009/2, N 190, 27-50. En ligne

16. https://journals.openedition.org/lhomme/22081?file=1].

17. Robert, M. (2013). Les Berbères et le Makhzen du Sud du Maroc: Essai sur la transformation politique des Berbères sédentaires (Groupe Chleuh). Beyrouth: Dar Al Aman.

18. Skali, F. (2006). Saints et sanctuaires de Fès. Rabat:Éditions Marsam. 